\section{GW23-e2647 STAGED HYBRID PROCEDURE FOR MYOCARDIAL REVASCULARISATION IN THE ELDERLY WITH CORONARY ARTERY DISEASE AND HIGH RISK STRATIFICATION}

doi:10.1136/heartjnl-2012-302920l.23

Yao Jianmin, Zhao Xiangdong, Lu Long, Wang Xian, Zhang Jian, Cui Zhentian, Liu Jing, Yao Jianmin. Department of Cardiovascular Surgery, General Hospital of Beijing Military Region, Beijing 100700, China

Objectives To summarise the experience of Staged hybrid procedure for Myocardial Revascularisation in the elderly with coronary artery disease and high risk stratification.

Methods Between August 2008 and December 2011, 10 elderly patients with coronary heart disease and high risk stratification, aged 60-75 years old, received staged hybrid procedure for Myocardial Revascularisation. Of the 10 patients, there was acute myocardial infarction (culprit lesion in circumflex or right coronary artery) concomitant with chronic total occlusion of left anterior descending artery in five cases. There was multivessel disease with bifurcation stenosis or calcification of left anterior descending artery and low left ventricular ejection fraction in 2, chronic obstructive pulmonary disease in 2 , chronic pericarditis in 1 . Offpump coronary artery bypass grafting was performed after percutaneous coronary intervention with drug-eluting stents for Myocardial Revascularisation.

Results All procedures were uneventful. The 10 patients received 13 stents, 15 grafts and 1 Balloon angioplasty. The average number of revascularisation was 2.9 per patient. Chest tube loss was 486 $\pm 172 \mathrm{ml}$, There was no perioperative myocardial infarction and inhospital death with a postoperative hospital-stay of $8.5 \pm 1.6$ days. During a follow-up of 2-28 months. There was no recurrent angina, late reintervention and mortality with New York Heart Association class I of cardiac function in 9, classIIin 1.

Conclusions Staged hybrid procedure seems a safe and effective alternative for myocardial revascularisation in the elderly with coronary artery disease and high risk stratification. The short-term results are satisfactory. The mid-term and long-term results need further investigation. 